\title{
DIE AKTUELLE LANDKARTE EIN NEUES GEOGRAPHISCHES INFORMATIONSMITTEL
}

Seit Anfang Januar 1954 erscheint im JRO-Verlag in München unter dem Titel «Die aktuelle JRO Landkarte» ein Unternehmen, das verdient, auch in unserem Lande und seinen Schulen bekannt gemacht zu werden. Von seinen Betreuern: dem Herausgeber Dr. ERnst Kremling und dem Redaktor Prof. Dr. Gustav Fochler-Hauke geschaffen, um einer weltweiten Orientierung über die derzeitigen Ereignisse in Nah und Fern zu dienen, hat es in den mehr als zwei Jahren des Erscheinens seinen Wert sehr eindrücklich unter Beweis gestellt. Das Kartenwerk bringt, vierzehntäglich in zwei Ausgaben, in einer Wandkarte $(116 \times 85 \mathrm{~cm})$ und einer Handkarte $(84 \times 62 \mathrm{~cm})$ erscheinend, jeweils eine globale Übersicht über die aktuellen Ereignisse in Kultur, Wirtschaft, Politik und Natur, wobei in den Mittelpunkt eine Erdkarte gestellt ist, um die sich mit Pfeillinien kurze Texte als Hinweise auf das Ereignis selbst gruppieren. Als Dominante des Kartenbildes figurieren zumeist Detaildarstellungen besonders aktueller Ereignisse einzelner Länder Ländergebiete oder der Gesamterde, mit denen nach Maßgabe der Aktualität abgewechselt wird. So entsteht eine zwanglose Folge von Karten, die insbesondere für Schulen jeder Stufe, Institute, wie auch für Betriebe höchst anregend und informativ zu wirken vermögen.

Dabei muß vor allem die Verbindung von globaler Gesamtübersicht und regionaler Detaildarstellung mit dem Mittel der thematischen physischen und Kultur- (Wirtschafts-, Bevölkerungs-, Verkehrs-) Karte als glücklich bezeichnet werden, da dadurch stets der Blick auf das Einzelne wie auf das Allgemeine gelenkt bleibt und damit in gewissem Sinne der Sinn für das Nationalbürgerliche und Weltbürgerliche zugleich geschult wird. 1)aß strenge Sachlichkeit sich bei allen Karten und Texten mit möglichster Anschaulichkeit part - es sind den Kartendarstellungen auch lehrreiche Zeichnungen Diagramme und Photos: z. B. von Städten, Heiligtümern, Industriegebieten usw. sowie statistische Tabellen beigegeben - ist bei einem solchen Unternehmen selbstverständlich. Der Wert des Ganzen wird überdies durch bibliographische Hinweise erhöht, die erkennen lassen, daß die Bearbeiter nicht nur neuste einheimische, sondern auch Quellen aus aller Welt benützen, so daß für Zuverlässigkeit des Dargebotenen gebürgt ist.

Eine Übersicht der uns vorliegenden Karten mag vom Reichtum der behandelten Bereiche überzeugen :

Globale Probleme: Die wichtigsten Weltereignisse der Jahre 1953, 1954, 1955. Islam (2), Französ. Union (4), Reis (8), Kohle: Weltreserven, Förderung, Ausfuhr, Verwertung (11), Erdöl (12), Katholizimus (14), Judentum (15), Vereinte Nationen (m.Triest, 16), Baumwolle (17), Eisenerzvorkommen : Reserven, Produktion (18), Intereuropäische, transozeanische und interkontinentale Bündnisse (19), Protestantismus (21), Volksdemokratische Staaten: Fläche, Bewohner, Wirtschaft (23), Heilige Stätten der Alten Welt (24), Bevölkerungsentwicklung und -kapazität (27), Uranerze und Atomwerke (29), Häfen und Schiffslinien (33), Staats- und Regierungsformen (34), Haustiere: Stammheimat, Wanderung, Nutzung (36), Großstädte (38), Volkseinkommen und Lebenshaltungskosten (42), Heer, Flotten, Luftwaffen (43), Sprachen (46), Kunst der Eiszeit (48), Getreide (55), Fischerei (56).

Europa: Europa seit 1945, Mittel- und Südosteuropa während des Zweiten Weltkrieges (5), Außenhandel der Bundesrepublik 1953 (12), Jugoslavien (26), Flüchtlinge in Deutschland (Europa und Asien 31), Österreich (37), Deutschland (Vom Kaiserreich zu den Republiken [39]), Europa (Politische und wirtschaftliche Zusammenarbeit, 40), Spanien (41), Saargebiet (44), Neugliederung der Bundesrepublik (51), Frankreich (53), Industriegebiete der Sowjetunion (52), Mitteldeutschland (54). Außereuropa: Afrika: Afrika seit 1945, Lybien (2), Großbritannien in Afrika (3), Marokko (4), Ägypten (13), Tunis (17), Sudan (45), Algerien (47); Amerika: Südamerika, ein Kontinent in Wandlung, Einwanderungsgebiet, Wirtschaftsunion Argentinien-Chile (7), Kanada (10), Guatemala (13), Brasilien (32); Asien: Indochina (4), Asien 1954 (8), Israel (15), Pakistan (20), Indische Union (22), Indonesien (28), Formosa, Ausbreitung des kommunistischen Machtbereichs in China 1945 bis 1955 (30), Japan (35), Cypern (50); Polargebiete: Nordpolargebiete (6), Antarktis (9).

Um darüber hinaus auch einen Begriff von den Einzeldarstellungen zu vermitteln, seien die Inhalte einiger Lieferungen angedeutet: So enthält die Kartennummer 26 Jugoslavien eine größere farbige Höhenschichtenkarte mit eingetragenen Wirtschaftszentren, Tabellen des Außenhandels, der Bergbauproduktion (1953), der Anbauflächen und Erträge, eine agrare Landnutzungskarte mit einem Anbauschema, eine Karte der Sprachen und Religionen, eine Lageskizze mit der Einordnung Jugoslaviens ins europäische Verkehrsnetz sowie die Hoheitszeichen und 2 Photos (Blick auf Split, Hüttenwerke in Zenika). Die Kartennummer 28 Indonesien umfasst eine Höhenschichtenkarte mit analogen wirtschaftlichen Signaturen, eine Karte der Verteilung des Waldes und Kulturlandes, eine Volksdichtekarte (in 5 Flächenstufen), Schiffahrtstraßen und Fluglinien, einen Größenvergleich mit Europa, eine Karte des Tenggergebirges, ein Höhenstufendiagramm, das Bild einer Reisbaulandschaft sowie verschiedene Tabellen und einen erläuternden Text, der sich geradezu als Lektionsskizze für den Lehrer der Volks- und Mittelschule verwenden ließe. Die Nummer 29 Uranerze und Atomkraftwerke, ein besonders aktuelles Thema, bringt eine Weltkarte mit den Uranlagerstätten (die nach ihrer Kapazität differenziert sind) und den Atomkraftwerken der verschiedenen Länder, eine Spezialkarte von Europa, Darstellung des Atombaus, das Schema eines Atomkraftwerks und ein analoges 
der Uranverarbeitung sowie eine gute Photo der (klassisch gewordenen) Atomwerke Oak Ridge in Tennessee und eine Photomontage des Atomwerkes von Dounreay in Schottland. Außerdem trägt zu deren Verständnis wiederum ein längerer erläuternder Text über Geschichte und gegenwärtige Situation der Atomkernenergiegewinnung mit reichen Literaturangaben wesentlich bei. In einer der jüngsten Nummern sodann, in Nr. 50 (25. Januar 1956) wird mit farbigen Karten von Cypern und Gaza die äusserst prekäre politische Situation in der östlichen Levante geschildert, wobei auch auf die bevölkerungspolitische und strategische Kapazität der arabischen Staaten und Israels hingewiẹsen wird. Nicht weniger instruktiv ist die hier als letzte zu erwähnende Nummer, die Frankreich gewidmet wurde und in Karten der Bevölkerungs- und Wirtschaftsstruktur, der Bevölkerungsdichte und -zunahme, der Wahlen der letzten Jahre, der Veränderungen im Kolonialreich sowie in graphischen Darstellungen der Lebenshaltung und des Sozialprodukts, der Lohnund Arbeitsverhältnisse ein sehr eindrückliches Bild der gegenwärtigen Tragik dieses Landes zeichnet.

So ließe sich für so gut wie jede Lieferung des Gesamtwerkes ein besonderer Reichtum an Tatsachen und eine individuelle Beleuchtung der in ihr enthaltenen "Welt" namhaft machen. Damit rechtfertigt sich für die bereits recht stattliche Kartenreihe das Prädikat eines Orbis pictus neuzeitlichen Gepräges, dem die Anerkennung weiter Kreise gebührt. Wir wünschen ihm auch an dieser Stelle Erfolg, und den Bearbeitern danken wir für ihre ausgezeichnete Idee und ihre Verwirklichung.

E. WINKLER

\section{DIE WELT IN DER WIR LEBEN \\ Zu einem Prachtwerk des a Life.}

Vor einigen Jahren unternahm die amerikanische Zeitschrift "Life im Rahmen anderer thematischer Darstellungen, ein Bild der Erde und ihrer Geschichte zu entwerfen, wie es bis dahin kaum je gewagt worden war. In einer Folge von zumeist farbigen Photos und Photomontagen realisierte sie in der Folge während der Jahre 1952-1955 ihren Plan, der offenbar im Leserkreis so großen Anklang fand, daß die Bild- und Textreihe auch als Buch herauskam. Nunmehr ist davon - im Th. Knaur Nachf. Verlag München bzw. im Buchclub Ex Libris Zürich - eine deutsche Ausgabe erschienen. Es darf wohl gesagt werden, daß dieses Werk - mit den Worten des diese deutsche Fassung einleitenden bekannten Zoologen Prof. Dr. HANS KRIEG - ein "großartiges Buch ist. Seine Fabel läßt sich zwar in wenige Symbole fassen: Geburt der Erde, Wunder und Rätsel des Meeres, Werden und Wandel des Festlandes, Lufthülle, Klima und Wetter, Zwei Milliarden Jahre Leben, Hohe Zeit der Säugetiere, Meeresleben, Das Korallenriff, Land unter der Sonne, Sieg des Lebens am Polarkreis, Tropenurwald, Sommergrüner Wald, Sternenströme im Kosmos, womit angedeutet ist, daß sie weder neu, originell noch konventionell ist. Was aber durchaus neu erscheint und faszinierend wirken muß auf jeden, dem realistisches Leben, realistisches Erdendasein noch etwas bietet, das ist die Farbenpracht der Landschaften der Gegenwart und ihrer Lebewesen, das ist die fugenlose Kombination von Photo und Typenbildern (Photomontage), die in der Tat, man mag über die Realistik oder Phantastik der letzteren denken, was man will, einen Kosmos vorzaubert, dem ein Alexander von Humboldo seinen Lobestribut nicht versagt hätte. Sicher werden die Rekonstruktionen der Dinosaurier-Landschaften und aller Lebensräume der Vorzeit phantastisch anmuten und manchem vielleicht die Lust nehmen, sich fürderhin in jene Epochen zurückzudenken. Die bei aller Farbigkeit der Bilder und Texte durchaus ernstsachliche Grundhaltung des Werkes jedoch - dem beste Namen amerikanischer und europäischer Forscher zu Pate standen, wie eine lange Dankesliste und eine nicht minder eingehende Bibliographie bezeugen, - wird auch für.jene geheimnisumwitterte Vorzeit die Vorstellung in éiner Weise klären, die überaus erhellend zu nennen ist. Mancher europäische Leser mag zwar beanstanden, daß die amerikanische Landschaft und ihre «amerikanische» Entwicklung als Beispiel erscheint, daß Europäisches allzusehr zurückgedrängt worden sei. Gerade die amerikanische Sicht wird indes dem wirklich Interessierten im besten Sinne informierend vorkommen, weil es zugleich im weitesten Sinne zu Vergleichen anregt und zu Vergleichen zwingt. Ganz gleich nun aber, ob "dieses Buch amerikanisch ist oder nicht, modern oder nicht, befremdend oder begeisternd; es ist ein Buch von großem Wurf, aufwühlend und bereichernd und von frischfröhlichem Zugriff, ein Weltbuch in doppeltem Sinne, handelnd von der Welt im Kosmos und sprechend zur Menschenwelt; ein Bilderbuch in des Wortes schönster Bedeutung... ein Buch für alle, ein Bilderbuch und ein Bildungsbuch zugleich» (KRIEG). Und für den Geographen bleibt nur ein Wunsch bestehen: daß ihm ein gleich Begeisterndes folgen möge, das auch die Welt des Menschen, Gegenwart und Entstehung der Kulturlandschaft und ihrer Mannigfaltigkeiten zeigen möge, das den «Kosmos» zum Ganzen fügt.

E. WINKLER

NACHTRAG ZU MEINER BESPRECHUNG DER NEUEN OBERSTUFENATLANTEN DES GEOGRAPHISCHEN INSTITUTES ED. HÖLZEI, IN WIEN IN DER GEOGRAPHICA HELVETICA, 1955, HEFT 4

Herr Dr. Hugo von Eckelt, der Inhaber und Leiter des Geographischen Institutes Ed. Hölzel in Wien, machte mir anläßlich einer kürzlichen Besprechung in Zürich folgende Mitteilungen : 